\title{
Serum Pooling Lowers Cholesterol Recovery ${ }^{1}$ )
}

\author{
Marcia Wills, Gregory Gerhardt, Mary Ann Holtz and Martin H. Kroll
}

Department of Pathology, Johns Hopkins School of Medicine, Baltimore, USA

\begin{abstract}
Summary: Matrix effects hinder the transferability of accuracy for cholesterol. A general assumption is that pooled and individual samples yield similar results. We tested the hypothesis that serum-pooling affects the recovery for cholesterol. We pooled 100 serum samples, determining cholesterol of pool and of the individual samples with Hitachi 717 and 914. Over twenty days, we daily determined cholesterol of individual and pooled samples, using a Hitachi 736 and 747 analyzers. For the hundred-sample pool, the pool was 1.1 to $1.5 \%$ lower than the individual samples. With the daily pool study, the ratio of 747 to 736 was $1.7 \%$ lower for the pooled compared with the individual samples.
\end{abstract}

Therefore, pooling of serum samples causes a decreased recovery, averaging from $1.1-1.7 \%$, and representing $37-57 \%$ of the allowable bias for cholesterol $(<3 \%)$, and it is thereby significant.

\section{Introduction}

The accuracy of cholesterol measurements is particularly important, because classification and management of lipid disorders is defined in absolute terms (1). Matrix effects hinder the transferability of accuracy (2). Altering the matrix of serum or plasma may have profound effects on the accuracy of methods for the determination of cholesterol (3-5). Lyophilization of sample pools can introduce these effects, therefore the use of fresh samples or pools of samples has been advocated and used to establish and maintain parity among analyzers $(5,6)$.

It has been assumed the pooling of samples does not alter the recovery of cholesterol. In our own laboratory program to maintain parity among analyzers, we measured the differences between our Hitachi 736 analyzer and Hitachi 747 analyzers for cholesterol. We found discrepancies for the degree of calibration bias between individual and pooled fresh samples. In this study, we examine the effects of pooling samples and transfering accuracy.

\section{Materials and Methods}

\section{Apparatus}

Boehringer Mannheim/Hitachi chemistry analyzers, models 736 , 747, 717, and 914 (Boehringer Mannheim Corporation, 9115 Hague Road, P. O. Box 50446, Indianapolis, IN 46250 USA). Two Hitachi 747 analyzers were used, one designated $747 \mathrm{~A}$ and the other 747B.

We determined cholesterol with the Cholesterol Method, Boehringer Mannheim/Hitachi (catalog number 1127578 for the Hitachi

\footnotetext{
1) Funding: Department of Pathology, Johns Hopkins School of Medicine
}

736 and 747 and 450026 for the Hitachi 717 and 914). The method uses cholesterol esterase ${ }^{2}$ ) to convert cholesterol esters to free cholesterol and fatty acids; cholesterol oxidase ${ }^{2}$ ) to produce unimolar amounts of cholest-4-en-3-one and hydrogen peroxide; and peroxidase $^{2}$ ) to convert the hydrogen peroxide with phenol and 4-aminoantipyrine to a quinone-imine dye, the latter used as the indicator for the reaction. For all four analyzers, we used the same calibrator (catalog number 620213 and lot number XLS-91).

\section{Procedures}

We studied the effect of pooling by comparing observed differences between analyzers using individual samples that were not part of the pool and by comparing pooled and individual samples that were part of the pool. All samples were sera, collected in gel collection tubes (Becton-Dickinson, Franklin, NJ, USA). All samples were stored refrigerated $\left(4-10^{\circ} \mathrm{C}\right)$. In the first study, over a twenty-day period, we daily made a ten-sample pool (taking one $\mathrm{ml}$ from each sample, mixing, and centrifuging to make the pool) and selected an individual sample, and in these samples we determined cholesterol. We used a new pool and individual sample each day.

We collected, over a period of seven days and then stored refrigerated, 100 serum samples with values between 4.92 and $5.44 \mathrm{mmol} / \mathrm{l}$ $(190$ to $210 \mathrm{mg} / \mathrm{dl})$ and made a large, amalgamated serum pool by aliquoting $0.1 \mathrm{ml}$ of serum from each individual sample. (The pipette used for making the pool was tested and found to have a precision error of $<0.2 \%$ of the deliverable volume.) We determined cholesterol of the pooled samples 200 times with the Hitachi 717 and 180 times with the Hitachi 914. The individual samples were determined in duplicate with both analyzers (except for one group of twenty samples that was run singly because of analyzer error). We interspersed each group of 20 individual samples with a group of 20 pooled samples, to minimize the effects of evaporation or drift. All samples were kept capped until they were placed and run on the analyzer. Because the variance of the pooled and individual samples is different, we compared the values with the Mann-Whitney test (two-tailed), a non-parametric test.

\section{2) Enzymes:}

Cholesterol esterase (microorganism): sterol esterase EC 3.1.1.13

Cholesterol oxidase (Nocardia erythropolis): cholesterol oxidase EC 1.1.3.6

Peroxidase (horseradish): peroxidase EC 1.11.1.7 
Over twenty days, we determined cholesterol for an individual sample with both the Hitachi 736 and 747 analyzers. We also determined cholesterol for a serum pool, made fresh daily (ten samples, mixed, and centrifuged), with both analyzers. We used a new individual specimen and pooled sample each day. By dividing the results from the Hitachi 747 analyzer by those of the Hitachi 736 , were derive an adjustment factor for the Hitachi 736 analyzer. Multiplying patient results produced by the Hitachi 736 by this adjustment factor should achieve parity between the two analyzers. It has always been assumed that one would obtain the same adjustment factor whether one used individual samples or pooled samples. We compared the means for the adjustment factors obtained with the different analyzers by Student's t-test.

\section{Results}

For the Hitachi 717 analyzer, the median cholesterol concentrations for the individual and amalgamated (100 specimens) serum pool samples were $5.206 \mathrm{mmol} / \mathrm{l}$ and $5.128 \mathrm{mmol} / \mathrm{l}$, respectively. Likewise, for the Hitachi 914 analyzer, the individual and pool medians were $4.921 \mathrm{mmol} / \mathrm{l}$ and $4.869 \mathrm{mmol} / \mathrm{l}$, respectively (tab. 1). The differences between these medians were 0.078 $\mathrm{mmol} / \mathrm{l}(1.5 \%)$ for the Hitachi 717 analyzer and 0.052 $\mathrm{mmol} / \mathrm{l}(1.1 \%)$ for the Hitachi 914 . These differences are statistically significant by the Mann-Whitney test, with the probability of the null hypothesis being less than 0.02 for both analyzers (tab. 1).

The distributions of the individual samples were symmetric, with skews of only 0.17 and -0.006 , for the Hitachi 914 and 717, respectively, indicating that these distributions were nearly Gaussian. The differences between the individual and pooled samples were $1.1 \%$ $(0.070 \mathrm{mmol} / \mathrm{l})$ for the Hitachi 717 and $0.94 \%$ (0.046 $\mathrm{mmol} / \mathrm{l}$ ) for the Hitachi 914 . The potential error from imprecise sampling can be estimated from the standard deviation of the individual samples and the pipetting error: $0.22 \times 0.002=0.00044$. This error divided by the

Tab. 1 Study of individual and pool sample descriptive statistics for cholesterol

\begin{tabular}{|c|c|c|c|c|}
\hline & \multicolumn{2}{|c|}{ Hitachi 717} & \multicolumn{2}{|l|}{ Hitachi 914} \\
\hline & Individual & Pool & Individual & Pool \\
\hline $\begin{array}{l}\text { Mean } \\
\quad(\mathrm{mmol} / \mathrm{l})\end{array}$ & 5.204 & 5.134 & 4.930 & 4.880 \\
\hline $\begin{array}{l}\text { Median } \\
(\mathrm{mmol} / \mathrm{l})\end{array}$ & 5.206 & 5.128 & 4.921 & 4.869 \\
\hline $\mathrm{N}$ & 200 & 200 & 180 & 180 \\
\hline $\begin{array}{l}\text { Variance } \\
\qquad\left(\mathrm{mmol}^{2} / 1^{2}\right)\end{array}$ & 0.045 & 0.0030 & 0.0486 & 0.0084 \\
\hline $\begin{array}{l}\text { Difference } \\
\qquad(\mathrm{mmol} / \mathrm{l})\end{array}$ & \multicolumn{2}{|c|}{0.070} & \multicolumn{2}{|c|}{0.050} \\
\hline $\begin{array}{l}\text { Standard error } \\
(\mathrm{mmol} / \mathrm{l})\end{array}$ & 0.0151 & 0.0039 & 0.0164 & 0.0068 \\
\hline Mann-Whitney $\mathrm{Z}$ & \multicolumn{2}{|c|}{-5.06} & \multicolumn{2}{|c|}{-2.5} \\
\hline Probability $\mathrm{H}_{\mathrm{o}}$ & \multicolumn{2}{|c|}{$4.3 \times 10^{-7}$} & \multicolumn{2}{|c|}{0.013} \\
\hline
\end{tabular}

difference renders the fraction the error contributes to the difference, $0.00044 \div 0.050=0.0088$, or $0.88 \%$, and thus it constitutes a small and inconsequential fraction of the total difference. Therefore, pipetting error could not distort the true concentration of the pool.

In the parity study for obtaining the adjustment factor, the individual samples ranged in cholesterol concentration from 2.253 to $12.044 \mathrm{mmol} / 1$ for the Hitachi 736 (mean \pm 1 SD: $5.517 \pm 2.497$ ) and 2.176 to 11.862 $\mathrm{mmol} / 1$ for the Hitachi 747 (mean $\pm 1 \mathrm{SD}$ : 5.361 $\pm 2.409 \mathrm{mmol} / \mathrm{l}$ ). For the pooled samples, over twenty days, the cholesterol concentration ranged from 2.875 to $6.397 \mathrm{mmol} / 1$ for the Hitachi 736 (mean \pm 1 SD: 4.533 $\pm 1.006 \mathrm{mmol} / \mathrm{l})$ and 2.720 to $6.527 \mathrm{mmol} / \mathrm{l}$ for the $\mathrm{Hi}$ tachi 747 (mean $\pm 1 \mathrm{SD}: 4.351 \pm 0.991 \mathrm{mmol} / \mathrm{l}$ ). The adjustment factor (mean Hitachi 747 value, the mean Hitachi 736 value) was $97.5 \%$ for the individual samples and $95.8 \%$ with the pooled samples. The pooled samples gave an adjustment factor $1.7 \%$ less than that obtained with the individual samples. This difference was statistically significant $\left(t=2.82, H_{o}: p<0.02\right)$.

\section{Discussion}

It has been a general assumption in lipid analysis, and most chemistry analyses as well, that when combining samples into a pool, the sum of values is equal to the whole. We expect the mean of a serum pool to equal to weighted mean from all individual samples.

The results demonstrate that for cholesterol recovery is lower for a pool rather than the individual samples, even when the pool is composed of individual samples and all determinations are done at about the same time. The decreased values for the pool could result from an altered configuration of the lipoproteins or the loss of lipoproteins in the mixing and centrifugation steps.

When one analyzer recovers differently from another analyzer with the same type of material, one must be cautious using the results to adjust the output of the former. Fresh, individual samples are the best material to use for establishing and maintaining parity, and the samples should be analyzed over multiple days. It is desirable that any other material, such as serum pools, should yield similar results to those of the individual samples.

In this study, we were adjusting the Hitachi 736 so it would yield results similar to those of the Hitachi 747 . The adjustment factor with fresh, individual samples was $97.5 \%$, however, the factor was $95.8 \%$ with pooled samples. The difference between these two factors is $1.7 \%$ and was statistically significant. This $1.7 \%$ difference is important because if one used the $95.8 \%$ value, from the pooled samples, one would lower the value resulted by the analyzer $1.7 \%$ below real patient sam- 
ples. This difference in the apparent accuracy represents $57 \%$ of the allowable error for cholesterol.

The recovery changes due to pooling most likely do not represent a loss of cholesterol in the sample, because nothing was removed from the samples during the pooling process and the two Hitachi 736 and 747 analyzers behaved differently with the pooled samples. The decreased recovery seen between the individual samples and the amalgamated pool may represent movement of cholesterol among lipoproteins from different samples, or a restructuring of the lipoprotein particles. Restructuring of the lipoproteins could include transfer of apolipoproteins within or between classes of particles, increased ratio of esterified to free cholesterol, or actual physicochemical changes such as an altered surface-ratio of phospholipids to cholesterol. Restructured lipoproteins can potentially alter the accessibility of cholesterol esterase or cholesterol oxidase to cholesterol esters or free cholesterol, respectively. The altered accessibility may require more time for either of the enzymatic reactions to reach endpoint. Decreased enzyme accessibility to substrate may decrease the rate of reaction and result in incomplete recovery (7). In an alternate mechanism, mixed lipoproteins from different patient sources may aggregate and precipitate out of solution.

In this study, the pooling of serum resulted in matrix effect of 1.1 to $1.7 \%$ decrease for serum cholesterol.

\section{References}

1. Current status of blood cholesterol measurement in clinical laboratories in the United States: a report from the Laboratory Standardization Panel of the National Cholesterol Education Program. Clin Chem 1988; 34:193-201.

2. Kroll MH, Lindsey H, Greene J, Sliva C, Hainline Jr A, Elin RJ. Bias between enzymatic methods and the reference method for cholesterol. Clin Chem 1988; 34:131-5.

3. Miller WG. Matrix effects in the measurement and standardization of lipids and apolipoproteins. Curr Opin Lipid 1992; 3:361-4.

4. Ross JW, Myers GL, Gilmore BF, Cooper GR, Naito HR, Eckfeldt J. Matrix effects and the accuracy of cholesterol analysis. Arch Pathol Lab Med 1993; 117:393-400.

5. Naito HK, Kwak Y-S, Hartfiel JL, Park JK, Travers EM, Myers GL, et al. Matrix effects on proficiency testing materials. Arch Pathol Lab Med 1993; 117:345-51.
Even though the magnitude of this difference may appear small, the allowed bias error for any field method is less than $3 \%$ from the reference method, as recommended by both Centers for Disease Control and National Cholesterol Education Program criteria. Frequently, one must use a serum pool to link one's results with the reference method, therefore, this bias error, even though appearing small, would interfere with the link between the reference method and the field method. The pooling effect in this study could contribute 37 to $57 \%$ of the error for allowable bias, sharply narrowing the tolerance for calibration bias. Our study suggests it is better to use individual samples to establish parity among analyzers and link field methods with the reference method. Individual samples have another advantage, in that the range of values is greater than with unadulterated sample pools and provide a better assessment of agreement.

\section{Acknowledgements}

The authors are grateful to the Clinical Chemistry Division, Johns Hopkins Outpatient Center, and Johns Hopkins at Greenspring Station Laboratory, all Divisions of the Department of Pathology of the Johns Hopkins Hospital, which together provided the cholesterol determinations. The authors thank Allen Valentine, Michael Huppenthal, Ellen Dumer, Ashraf H. Fatani, and Robin Barr for their organization and technical assistance.

6. Kroll MH, Chesler R, Elin RJ. Effect of lyophilization on results of five enzyme methods for cholesterol. Clin Chem 1989; 35:1523-6.

7. Tel RM, Berends GT. Incomplete hydrolysis of cholesteryl esters during the enzymatic cholesterol determination as evidence by aqueous cholesterol ester solutions: comparison of six enzymatic procedures with the Liebermann-Burchard method. J Clin Chem Clin Biochem 1980; 18:595-601.

\section{Received February 10/April 28, 1997}

Corresponding author: Martin H. Kroll, MD, Johns Hopkins School of Medicine, Department of Pathology, 600 North Wolfe Street, Meyer B-125, Baltimore, MD 21287, USA

Fax: +1-410/955-0767, e-mail: mkroll@pathlan.path.jhu.edu 

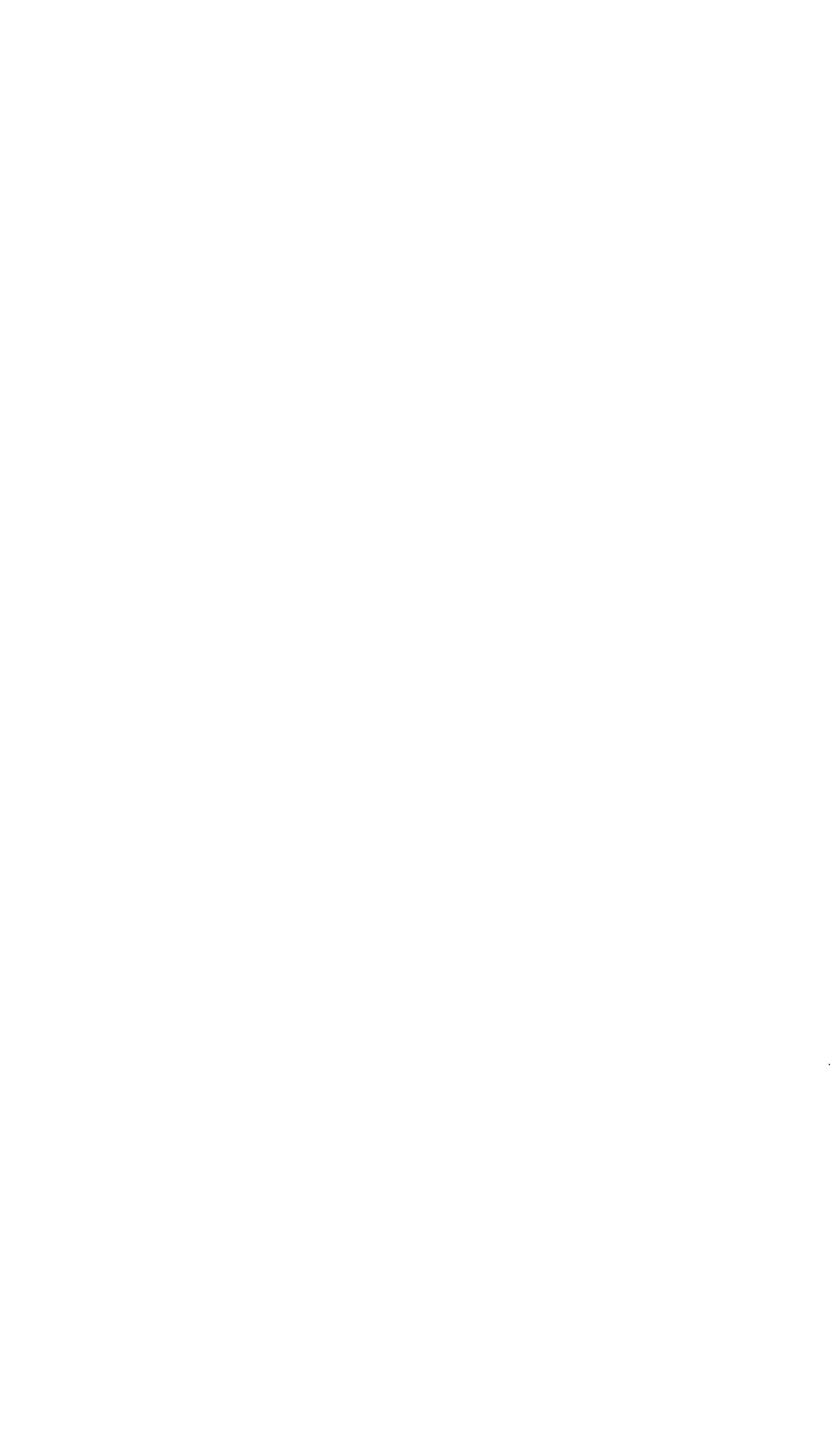